\title{
Development and Validation of Prognostic Nomograms for Elderly Patients with Osteosarcoma
}

\author{
Xiaoqiang Liu' \\ Shaoya $\mathrm{He}^{2}$ \\ Xi Yao' \\ Tianyang $\mathrm{Hu}^{3}$ \\ 'Department of Orthopedic Surgery, \\ Anyue County People's Hospital, Sichuan, \\ People's Republic of China; ${ }^{2}$ Department \\ of Gastroenterology, Anyue County \\ People's Hospital, Sichuan, People's \\ Republic of China; ${ }^{3}$ Department of \\ Cardiology, The Second Affiliated \\ Hospital of Chongqing Medical University, \\ Chongqing, People's Republic of China
}

Correspondence: Tianyang Hu Department of Cardiology, The Second Affiliated Hospital of Chongqing Medical University, 74 Linjiang Road, Yuzhong District, Chongqing, 4000I0, People's Republic of China

Tel +8623-63693222

Email hutianyang@stu.cqmu.edu.cn
Background: The aim of the current study was to construct prognostic nomograms for individual risk prediction in elderly patients with osteosarcoma.

Methods: Data for 816 elderly patients ( $\geq 40$ years old) with osteosarcoma between 2004 and 2016 from the Surveillance, Epidemiology, and End Results (SEER) database were randomly assigned to training $(\mathrm{N}=573)$ and internal validation $(\mathrm{N}=243)$ sets. The essential clinical predictors were identified based on least absolute shrinkage and selection operator (Lasso) Cox regression. Nomograms were constructed to predict the 1-, 3-, and 5-year cancer-specific survival (CSS) and overall survival (OS).

Results: Our LASSO regression analyses of the training set yielded five clinicopathological features (age, chemotherapy, surgery, AJCC stage, and summary stage) in the training cohort for the prognosis of elderly patients with osteosarcoma, while grade was only associated with OS and M stage was only associated with CSS. Construction of nomograms based on these predictors was performed to evaluate the prognosis of elderly patients with osteosarcoma. The C-index, calibration and decision curve analysis also showed the satisfactory performance of these nomograms for prognosis prediction.

Conclusion: The constructed nomograms are helpful tools for exactly predicting the prognosis of elderly patients with osteosarcoma, which could enable patients to be more accurately managed in clinical practice.

Keywords: osteosarcoma, elderly, SEER, nomograms, prognosis, risk management

\section{Introduction}

Osteosarcoma, which is particularly prevalent in children, adolescents, and young adults, is one of the most lethal bone malignant tumors with an aggressive clinical behavior and poor prognosis. ${ }^{1-3}$ Due to their different gene expression, therapeutic response, and their heterogeneous histological subtypes, osteosarcoma can be diagnostically and prognostically challenging. ${ }^{4-6}$ Moreover, patients with osteosarcoma older than 40 years old exhibit a lower survival rate than those of adolescents even though multidisciplinary treatment including surgery and chemotherapy. ${ }^{7-9}$ Thus, identification of older patients with osteosarcoma who are at high risk of mortality can ensure the implementation of appropriate treatment and have a substantial impact on prognosis.

As a statistical predicted model, the nomogram represents a schema of diagram, in which variables are given scores, and hence it's readily accessible for the probability of an event for an individual patient, in comparison with conventional assessment criteria. ${ }^{10}$ 
In recent years, this model has been widely applied as the increased need of individualized medicine in a great variety of malignant tumors as well as in patients with different types of osteosarcoma. ${ }^{11-15}$ Since different clinical and prognosis features between two age groups, no study is available to develop a prognostic nomogram for osteosarcoma in elderly patients. Therefore, in this study, we aimed to construct and verify a survival nomogram integrating the accessible clinical features to improve prognostication for elderly patients osteosarcoma in clinical practice.

\section{Methods}

\section{Study Population}

In this retrospective study, elderly patients ( $\geq 40$ years old) newly diagnosed with osteosarcoma by positive histology from the year of 2004-2016 according to "International Classification of Diseases for Oncology, 3rd Edition (ICDO-3) Hist/bahav" (9180/3, Osteosarcoma, NOS, 9181/3, Chondroblastic osteosarcoma, 9182/3, Fibroblastic osteosarcoma, 9183/3, Telangiectatic osteosarcoma, 9184/3, Osteosarcoma in Paget disease of bone, 9185/3, Small cell osteosarcoma, 9186/3, Central osteosarcoma, 9187/3, Intraosseous well differentiated osteosarcoma, 9192/3, Parosteal osteosarcoma, 9193/3, Periosteal osteosarcoma and 9194/3, High grade surface osteosarcoma). Methods of data collection and follow-up of patients are available on the SEER database. We further excluded patients with a history of other malignancies and unknown variables, such as race/ethnicity, age of diagnosis, TNM stage, American Joint Committee on Cancer (AJCC stage), SEER summary stage, marital status, tumor size, survival months. Based on the aforementioned selection, there were 816 cases left in this study. All of the patients included in this study were randomly assigned to the training group and validation group according to the ratio of 7:3 (training cohort, $\mathrm{n}=573$; validation cohort, $\mathrm{n}=243$ ).

\section{Variables}

The following variables were identified from the dataset: year of diagnosis (2004-2008, 2009-2012, 2013-2016), age at diagnosis ( $<60$ or $\geq 60$ years), race (white, black, and others), marital status (married or unmarried), primary site (bones and joints or others), Laterality (left, right or bilateral/others) grade (I/II or II/ IV), SEER summary stage (localized, regional or distant), tumor size $(<5,5-10$ or $>10 \mathrm{~cm})$, AJCC stage (I/II or III/ IV), T stage (T1, T2 or T3), N stage (N0 or N1), M stage
(M0 or M1), surgery for primary site (yes or no/unknown), chemotherapy (yes or no/unknown), radiotherapy (yes or no/unknown). The primary endpoint outcome of this study was OS while the secondary outcome was cancer-specific survival (CSS). Approval for this study was obtained from the Ethics Committee of Anyue County People's Hospital. Authors have signed the data-use agreement and got permission from SEER program to use this data.

\section{Statistical Analysis}

Count and percentage were used to describe categorical measurements and mean and range were applied to present continuous measurements. The chi-square test was used to compare the categorical measurements, while $t$-test or Mann-Whitney $U$-test was used to compare continuous variables. We used the least absolute shrinkage and selection operator (Lasso) Cox regression model to identify independent risk factors for CSS and OS. Compared with the traditional stepwise Cox regression analysis, Lasso Cox regression can reduce the estimation variance while providing an interpretable final model, which may more accurate than stepwise selection. ${ }^{16}$ To provide clinicians with a measurable instrument to predict the mortality of individual patients, we built two nomograms on the basis of Cox analysis in the training cohort. The time-dependent receiver operating characteristic curve (tdROC), calibration curve and decision curve analysis (DCA) were calculated to evaluate the predictive performance of prognostic nomograms. All statistical analyses were performed using $\mathrm{R}$ (version 3.4.3; www.r-project.org). $\mathrm{P}$ value $<0.05$ at two sides was defined as statistically significant. SEER publicuse research data from 2004 to 2016 were obtained from the SEER website (April 2019 release) and were based on the November 2018 submission.

\section{Results \\ Patients Characteristics}

From 2004 to 2016, a total of 816 elderly osteosarcoma patients (573 patients in the training set and 273 patients in the validation set) were finally included in this study. The mean age of elderly osteosarcoma patients was $58.7 \pm 13.1$ (years) in the training set, $59.4 \pm 14.0$ (years) in the validation set, and no significant differences were observed among the two sets as detected by analysis of variance $(\mathrm{P}=0.503)$. As shown in Table 1, there were no significant differences were observed among the two sets as detected by analysis of variance (all $\mathrm{P}>0.05$ ). Moreover, the median survival time was 
Table I Clinicopathological Characteristics of All Patients

\begin{tabular}{|c|c|c|c|}
\hline Characteristics & Training Set $(n=573)$ & Validation Set $(n=243)$ & $P$ value \\
\hline Year of diagnosis, $n$ (\%) & & & 0.858 \\
\hline 2004-2008 & $210(36.6)$ & $83(34.1)$ & \\
\hline $2009-2012$ & $182(31.8)$ & $92(37.9)$ & \\
\hline $2013-2016$ & 181 (31.6) & $68(28.0)$ & \\
\hline Age (years), n (\%) & & & 0.869 \\
\hline$<60$ & $329(57.4)$ & $138(56.8)$ & \\
\hline$\geq 60$ & $244(42.6)$ & $105(43.2)$ & \\
\hline Sex, male, $n(\%)$ & $303(52.9)$ & $120(49.4)$ & 0.361 \\
\hline Race, n (\%) & & & 0.557 \\
\hline White & $442(77.1)$ & $187(77.0)$ & \\
\hline Black & $87(15.2)$ & $31(12.8)$ & \\
\hline Others & $44(7.7)$ & $25(10.2)$ & \\
\hline Marital status, $\mathrm{n}(\%)$ & & & 0.365 \\
\hline Married & 327 (57.1) & $147(60.5)$ & \\
\hline Unmarried & $246(42.9)$ & $96(39.5)$ & \\
\hline Primary site, n (\%) & & & 0.053 \\
\hline Bones and joints & $480(83.8)$ & 191 (78.6) & \\
\hline Others & $93(16.2)$ & $52(21.4)$ & \\
\hline Laterality, n (\%) & & & 0.826 \\
\hline Left & $209(36.5)$ & $80(32.9)$ & \\
\hline Right & $212(37.0)$ & $104(42.8)$ & \\
\hline Bilateral/others & $152(26.5)$ & $59(24.3)$ & \\
\hline Histological grade, n (\%) & & & 0.061 \\
\hline Grade I/II & 215 & $73(30.0)$ & \\
\hline Grade III/IV & $358(62.5)$ & $170(70.0)$ & \\
\hline Summary stage, $n(\%)$ & & & 0.076 \\
\hline Localized & $24 \mid(42.1)$ & $92(37.9)$ & \\
\hline Regional & $214(37.3)$ & $85(35.0)$ & \\
\hline Distant & $118(20.6)$ & $66(27.1)$ & \\
\hline Tumor size, n (\%) & & & 0.376 \\
\hline$<5 \mathrm{~cm}$ & $217(37.9)$ & $88(36.2)$ & \\
\hline $5-10 \mathrm{~cm}$ & $209(36.5)$ & $83(34.2)$ & \\
\hline$>10 \mathrm{~cm}$ & $147(25.7)$ & $72(29.6)$ & \\
\hline AJCC stage, $n(\%)$ & & & 0.136 \\
\hline $1 / / 1$ & $419(73.1)$ & $164(67.5)$ & \\
\hline $\mathrm{III/IV}$ & $154(26.9)$ & $79(32.5)$ & \\
\hline T stage, $n(\%)$ & & & 0.076 \\
\hline $\mathrm{TI}$ & $292(51.0)$ & III (45.7) & \\
\hline $\mathrm{T} 2$ & $247(43.1)$ & $110(45.3)$ & \\
\hline $\mathrm{T} 3$ & $34(5.9)$ & $22(9.0)$ & \\
\hline $\mathrm{N}$ stage, $\mathrm{n}(\%)$ & & & 0.192 \\
\hline No & $522(91.1)$ & $228(93.8)$ & \\
\hline $\mathrm{NI}$ & $51(8.9)$ & $15(6.2)$ & \\
\hline M stage, $n$ (\%) & & & 0.185 \\
\hline Mo & $465(81.2)$ & $187(77.0)$ & \\
\hline MI & $108(18.8)$ & $56(23.0)$ & \\
\hline Chemotherapy, n (\%) & & & 0.415 \\
\hline Yes & $322(56.2)$ & $129(53.1)$ & \\
\hline No/unknown & $251(43.8)$ & $114(53.1)$ & \\
\hline
\end{tabular}


Table I (Continued).

\begin{tabular}{|l|l|l|l|}
\hline Characteristics & Training Set $(\mathbf{n}=\mathbf{5 7 3})$ & Validation Set $(\mathbf{n = 2 4 3 )}$ & P value \\
\hline $\begin{array}{l}\text { Surgery for primary site, } \mathrm{n}(\%) \\
\text { Yes } \\
\text { No/unknown }\end{array}$ & $\begin{array}{l}441(77.0) \\
132(23.0)\end{array}$ & $\begin{array}{l}187(77.0) \\
56(23.0)\end{array}$ & 0.998 \\
\hline $\begin{array}{l}\text { Radiation, } \mathrm{n}(\%) \\
\text { Yes } \\
\text { No/unknown }\end{array}$ & $117(79.6)$ & $\begin{array}{l}53(21.8) \\
190(78.2)\end{array}$ & 0.655 \\
\hline Survival months, M (1/4, 3/4) & $456(79.6)$ & $19.0(6.0,56.0)$ & 0.579 \\
\hline OS, $\mathrm{n}(\%)$ & $20.0(7.5,55.0)$ & $136(56.0)$ & 0.938 \\
\hline CSS, $\mathrm{n}(\%)$ & $319(55.7)$ & $114(46.9)$ & 0.885 \\
\hline
\end{tabular}

$20.0(7.5,55.0)$ months in the training set and $19.0(6.0,56.0)$ months in the validation set. Other clinical and pathological features are listed in Table 1.

\section{Construction and Verification of the OS Nomogram}

Based on the results of LASSO regression (Figure 1A and B), six features (age, tumor grade, chemotherapy, surgery primary site, AJCC stage, and summary stage) with nonzero coefficients were finally incorporated into the development
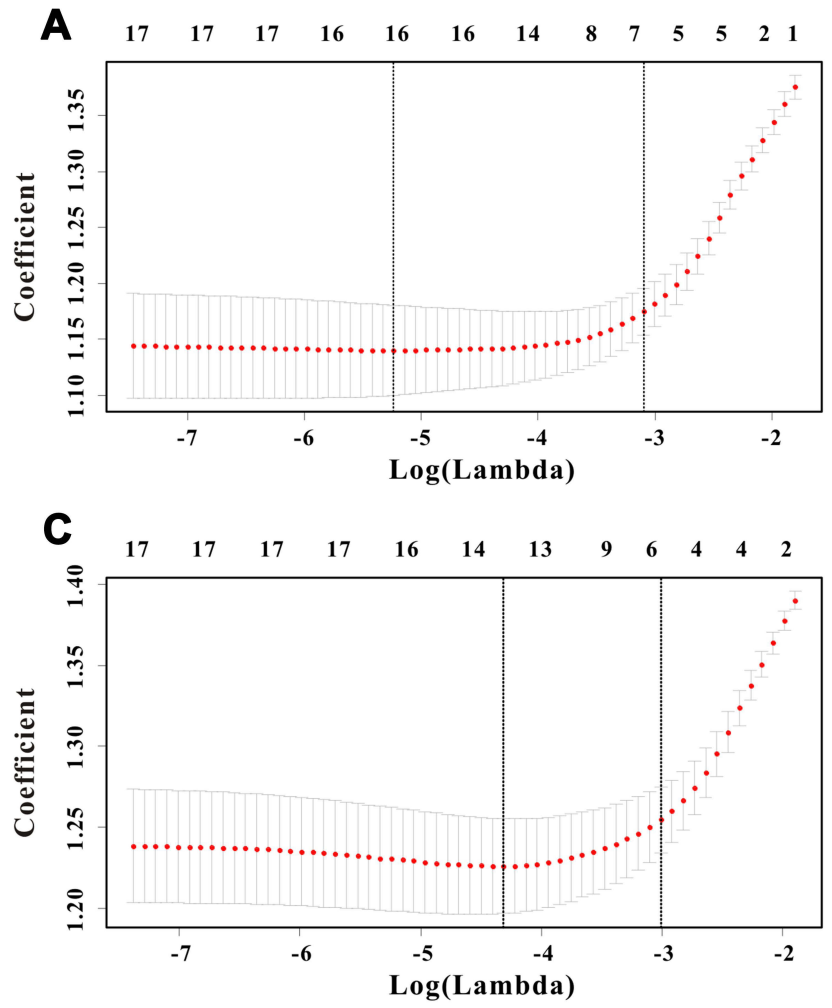

of a survival nomogram in the training set. The $\mathrm{C}$-index of the nomogram for OS in the training set and in the validation set was $0.774,0.815$, respectively. Moreover, as displayed in Figure 2A, this survival nomogram was very intuitive to predict the 1-year, 3-year and 5-year OS rates of patients with osteosarcoma. Figure $3 \mathrm{~A}-\mathrm{C}$ exhibited the calibration curves of the survival nomogram; plots were very close to the 45-degree line, indicating that the survival nomogram was well-calibrated in the training set. More specifically, tdROC analyses (Figure 4A) revealed that the survival nomogram could accurately predicted the 1-year (AUC=0.819), 3-year
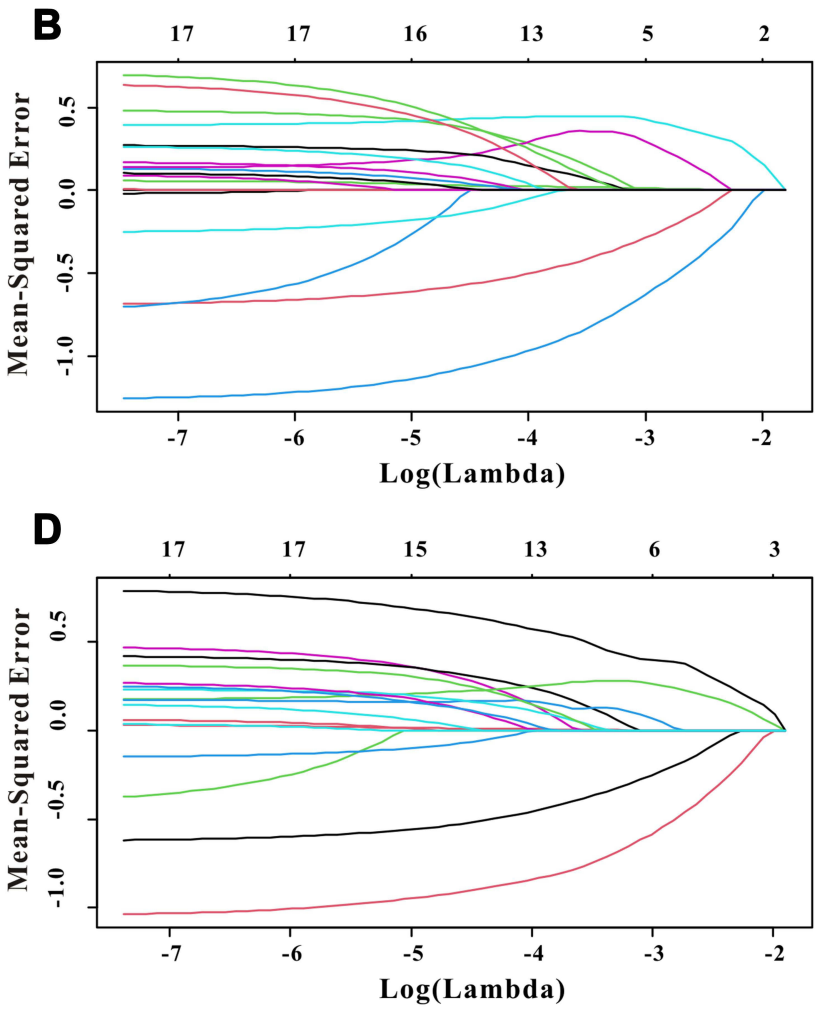

Figure I Selection of informative factors associated with OS and CSS using the LASSO Cox regression model. (A) LASSO coefficient profiles of all clinical features for OS. (B) Selection of the tuning parameter ( $\lambda$ ) for OS. (C) LASSO coefficient profiles of all clinical features for CSS. (D) Selection of the tuning parameter ( $\lambda$ ) for CSS. 
A

Points

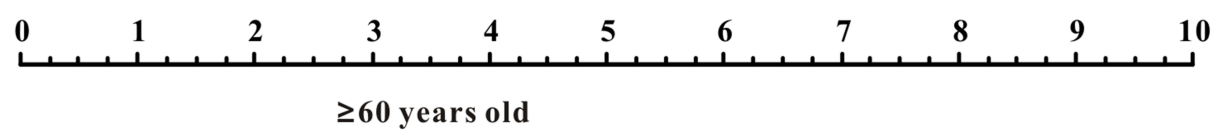

Age

Chemotherapy

Grade

AJCC stage

Sugery

Summary stage

Total Points

1-year OS

3-year OS

5-year OS
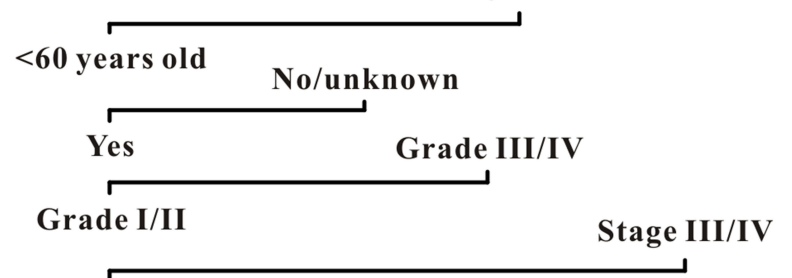

Stage I/II No/unknown

-year OS

B

Points

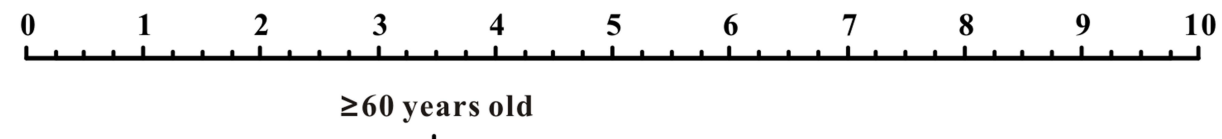

Age

Chemotherapy

M stage

AJCC stage

$<60$ years old

$$
0
$$

10

15

Regional

M0 M1

Localized Distant

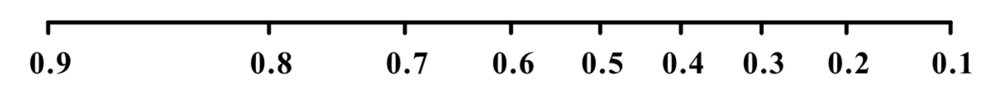

$\begin{array}{lllllll}0.7 & 0.6 & 0.5 & 0.4 & 0.3 & 0.2 & 0.1\end{array}$

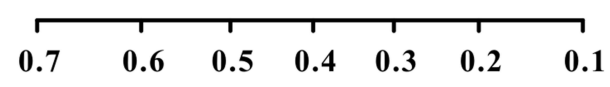

Sugery

Summary stage
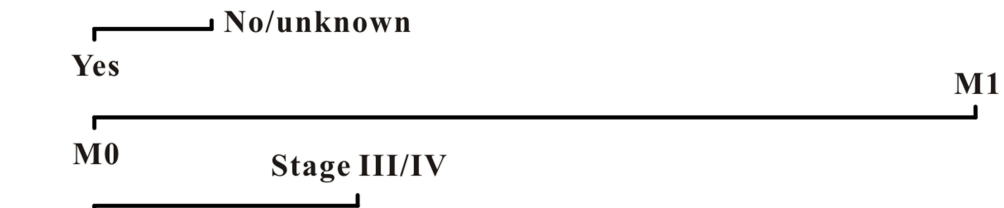

Stage I/II

No/unknown

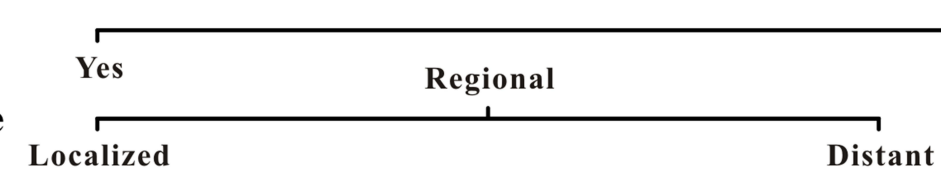

Total Points

1-year CSS

\begin{tabular}{lllllllll}
\hline 0 & 5 & 10 & 15 & 20 & 25 & 30 & 35 & 40
\end{tabular}

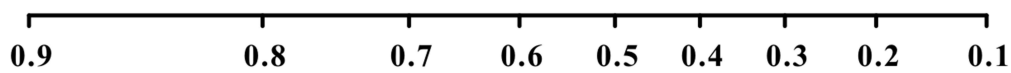

3-year CSS

5-year CSS
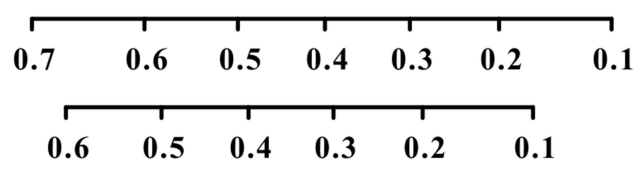

Figure 2 Survival nomogram for the prediction of 3-year, 5-year and 10-year OS (A) and CSS (B) in elderly osteosarcoma patients. 

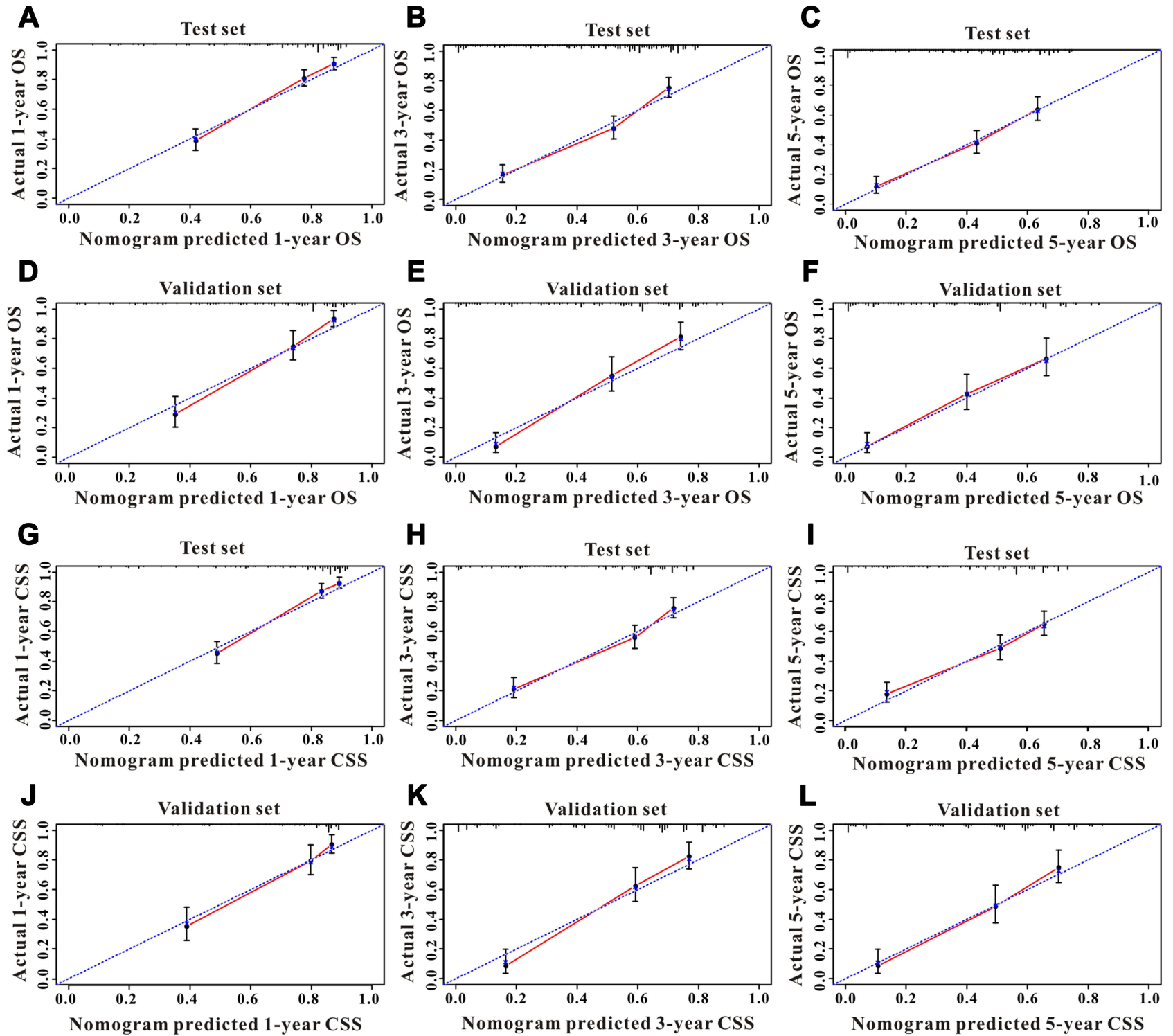

Figure 3 The calibration curves for predicting OS and CSS in the training and validation sets. Calibration plots of I-year, 3-year and 5-year OS in the training cohort (A-C) and in the SEER validation cohort (D-F). Calibration plots of I-year, 3-year and 5-year CSS in the training cohort (G-I) and in the SEER validation cohort (J-L).

$(\mathrm{AUC}=0.758)$ and 5 -year $(\mathrm{AUC}=0.746)$ survival rates in patients with osteosarcoma. DCA curve also demonstrated that the survival nomogram derived from the training set was clinically useful (Figure 5A).

To evaluate the calibration of the survival nomogram, we compared the predicted 1-year, 3-year, and 5-year survival probabilities with the correspondingly actual observations. As shown in Figure 3D-F, the calibration curves of the survival nomogram exhibited good concordance between the predicted probabilities and actual outcomes. Similarly, tdROC curves (Figure 4B) displayed that the survival nomogram possessed excellent predictive performances for 1-year, 3 -year, and 5-year survival, as reflected by an AUC of 0.837 ,
0.830 and 0.772 , respectively. Additionally, our DCA curve from the validation set also proved that the survival nomogram was clinical utility (Figure 5B).

\section{Construction and Verification of the CSS Nomogram}

Based on the results of LASSO regression (Figure 1C and D), six features (age, chemotherapy, surgery primary site, AJCC stage, M stage, and summary stage) with nonzero coefficients were finally incorporated into the development of a CSS nomogram in the training set. The C-index of the nomogram for CSS in the training set and in the validation set was $0.752,0.791$, respectively. Moreover, as displayed 

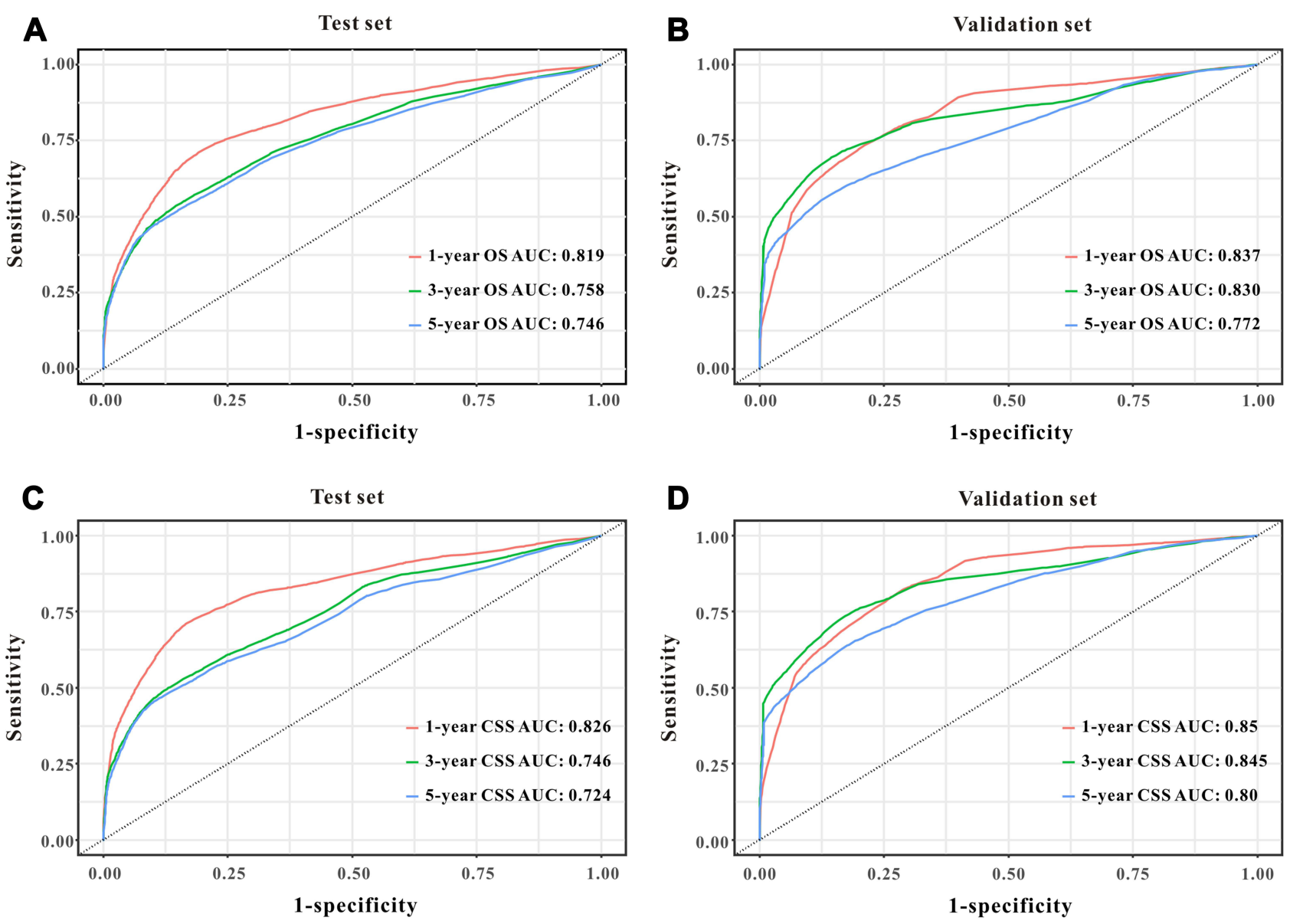

Figure 4 Predictive performance of the survival nomogram reflected by td-ROC curves. td-ROC curves for the I-year, 3-year and 5-year OS in patients in the training cohort (A) and in the validation cohort (B). td-ROC curves for the 1-year, 3-year and 5-year CSSS in patients in the training cohort (C) and in the validation cohort (D).

in Figure 2B, each subtype within these covariates was assigned a point on the point scale. By adding the total points together and locating it on the bottom scale, we were able to calculate the probability of 3-, 5- and 10-year cancer-specific and overall survival. As displayed in Figure $3 \mathrm{G}-\mathrm{I}$, the actual and the predicted probability of CSS for 1, 3 and 5 years between training set were in good agreement. Hence, the nomograms were well calibrated in the training set. Moreover, tdROC analyses (Figure 4C) revealed that the survival nomogram could accurately predict the 1-year $(\mathrm{AUC}=0.826), 3$-year (AUC $=0.746)$, and 5-year $(\mathrm{AUC}=0.724)$ survival rates in patients with osteosarcoma. DCA curve also demonstrated that the survival nomogram derived from the SEER training set was clinically useful (Figure 5C).

As shown in Figure 3J-L, the calibration curves of the survival nomogram exhibited good concordance between the predicted probabilities and actual outcomes. Similarly,
tdROC curves (Figure 4D) displayed that the survival nomogram possessed great predictive performances for 1-, 3- and 5-year survival, as shown in an AUC of 0.850 , 0.845 and 0.800 , respectively, in the validation set. To evaluate the calibration of the survival nomogram, we compared the predicted 1-year, 3-year and 5-year survival probabilities with the correspondingly actual observations. Additionally, our DCA curve from the validation set also proved that the survival nomogram was clinical utility (Figure 5D).

Kaplan-Meier curves of overall survival for patients stratified by the tertile of total points predicted by the nomograms in training cohort (Figure 6A) and in the validation cohort (Figure 6B). Kaplan-Meier curves of cancer-specific survival for patients stratified by the tertile of total points predicted by the nomograms in training cohort (Figure 6C) and in the validation cohort (Figure 6D). 

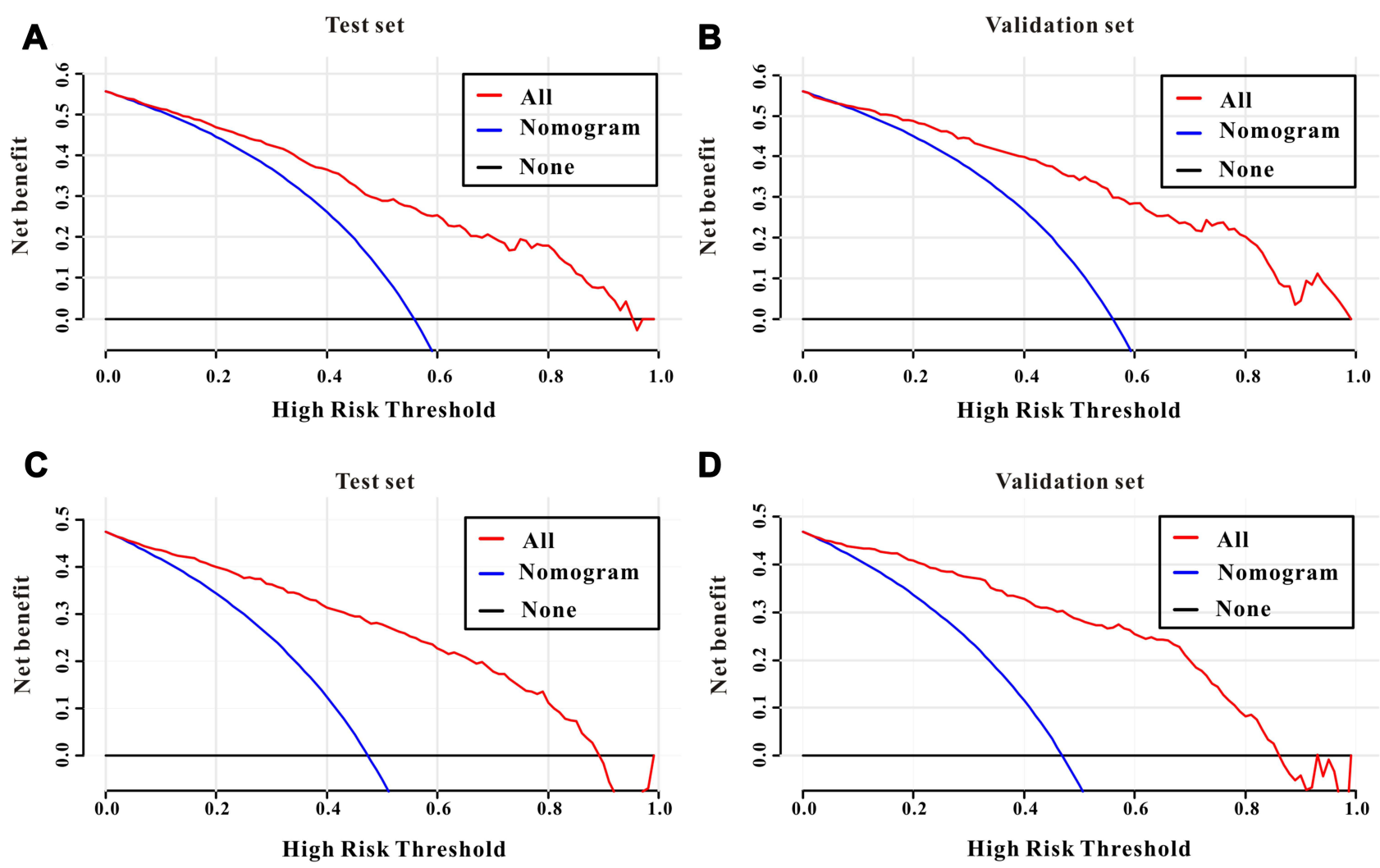

D

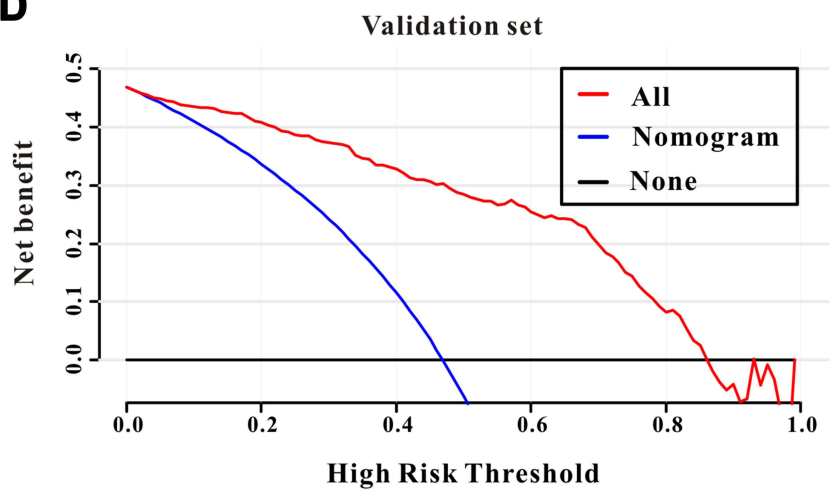

Figure 5 Decision curves analysis (DCA) for the survival nomogram to assess its clinical usefulness. The DCA of survival nomogram for OS in the training (A) and in the validation sets (B). The DCA of survival nomogram for CSS in the training (C) and in the validation sets (D).

\section{Discussion}

Several prognostic factors are associated with mortality for elderly patients with osteosarcoma in previous studies. However, the single factor can only achieve finite accuracy of predicting patients' prognosis. Hence, in the current study, our study firstly developed and verified two nomograms based on a variety of clinical and pathological features to predict the 1-year, 3-year and 5-year OS and CSS in elderly patients with osteosarcoma. Our survival nomograms showed great predictive performance. This forcefully indicated that our survival nomograms might be a useful tool for individual elderly osteosarcoma patient survival estimation.

Nomogram, an easy-to-use comprehensive tool, which could incorporate the conducive factors together, is of great significance for clinicians to accurately clarify diagnosis and predict survival of individual patient. ${ }^{17-20}$ As for patients with osteosarcoma, several predictive models have been constructed in previous studies, ${ }^{6,11,13}$ however, limited data is available for this special age group. Besides, independent predictors for OS and CSS in elderly patients with osteosarcoma are still controversial until now. For the aforementioned reasons, we aimed to develop and validate two nomograms with high-efficiency and high-accuracy for OS and CSS for individual patient with osteosarcoma. Base on Lasso Cox regression, six informative variables (age, tumor grade, AJCC stage, SEER summary stage, surgery and chemotherapy) were determined as prognostic factors for OS while age, $M$ stage, AJCC stage, SEER summary stage, surgery and chemotherapy were selected for CSS in elderly patients with osteosarcoma.

Age is a well-known prognostic factor for many tumors. ${ }^{21-23}$ In patients with osteosarcoma, Hagleitner et al demonstrated that the 5-year OS rate for patients aged $20-40$ years was $33.3 \%$ while $70.6 \%$ in $\leq 14$ years old and $52.5 \%$ in $15-19$ years old in a retrospective study of 102 osteosarcoma patients. ${ }^{24}$ Moreover, Tsuchie et al compared the prognosis of primary osteosarcoma in younger and older patients based on a cut-off age of 40 and concluded that the older patients showed a poorer prognosis. ${ }^{8}$ Consisting with these studies, age also an independent factor for OS as well as CSS for patients 
A

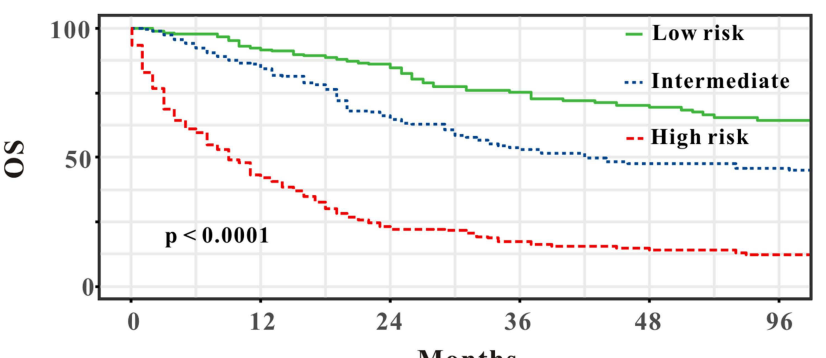

B Validation set

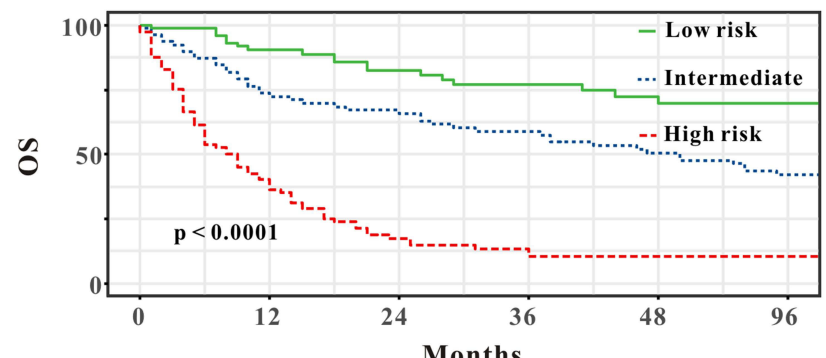

Number at risk Months

$\begin{array}{lrcccc}\text { Low risk } & 178 & 152 & 126 & 95 & 77 \\ \text { Intermediate186 } & 146 & 98 & 70 & 60 \\ \text { High risk } & 209 & 83 & 41 & 26 & 20\end{array}$

\section{Number at risk}

Months

\begin{tabular}{llrlllll}
\multicolumn{2}{l}{ Number at risk } & & & & \\
61 & Low risk & 82 & 62 & 49 & 38 & 28 & 20 \\
52 & Intermediate 80 & 55 & 49 & 43 & 36 & 29 \\
14 & High risk & 81 & 32 & 13 & 9 & 7 & 6
\end{tabular}

c

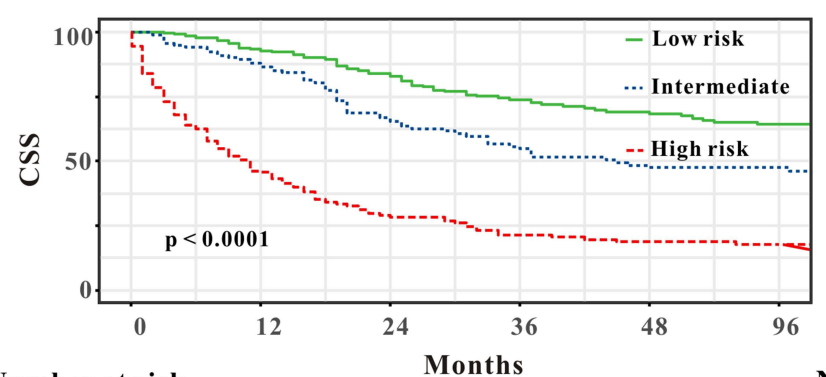

Number at risk

Low risk $\mathbf{2 2 2}$

Intermediate158

High risk 193
185

122

74
Test set

D
Validation set

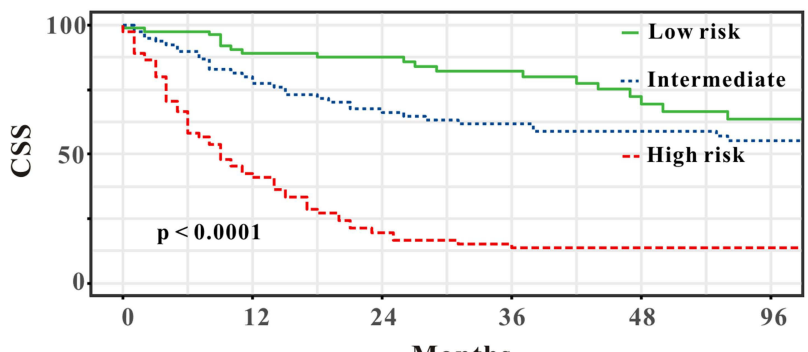

Number at risk

Months

$\begin{array}{lllll}62 & 51 & 39 & 26 & 18 \\ 58 & 47 & 41 & 37 & 30 \\ 29 & 13 & 10 & 8 & 7\end{array}$

Figure 6 Analysis of the prognostic significance of the nomogram in elderly osteosarcoma patients. Kaplan-Meier curves of OS for all patients stratified by the tertiles points predicted by the nomograms in training cohort $(\mathbf{A})$ and in the validation set (B). Kaplan-Meier curves of CSS for all patients stratified by the tertiles points predicted by the nomograms in training cohort (C) and in the validation set (D).

with osteosarcoma based on Lasso COX regression. Several reasons may lead to this result, including the delayed medical advice, resistance to chemotherapy, low tolerance for risky surgery, and more metastasis at diagnosis. ${ }^{7,25}$ In previous studies, osteosarcoma patients with distant metastatic at diagnosis had a relatively higher mortality compared to those with local/regional disease. $^{26,27}$ In the current study, both SEER summary stage and AJCC stage were determined for OS as well as CSS by Lasso COX regression which indicated that both the regional and distant metastasis were associated with a higher risk of mortality after adjusting for the confounding factors. Unlike some other solid malignant tumors, the benefit of chemotherapy for osteosarcoma patients is still controversial. $^{28-30}$ Fan et al conducted a meta-analysis based on the data of seven randomized controlled trials and concluded that ifosfamide-based chemotherapy can significantly improve event-free survival and OS. ${ }^{31}$ However, a recent study of 736 adolescents with juvenile osteosarcoma from the SEER database demonstrated that the treatment of chemotherapy did not associate with improved OS as well as $\mathrm{CSS}^{32}$ In our study, we found that the elderly osteosarcoma patients without chemotherapy had an unfavorable prognosis, which was in accordance with previous studies for older patients. ${ }^{33,34}$ Surgical resection of primary site plays a pivotal role in the management of patients with osteosarcoma. In our study, we demonstrated that elderly patients with surgery resection had an advantageous survival rate. In addition, the detailed information on chemotherapy and surgical margins did not accessible for SEER database, thus we could not further explore the role of those two variables.

The feasible limitations should be considered in this study. Firstly, since the SEER database provides limited information, we did not analyze laboratory test indicators or detailed treatment information, such as specific chemotherapy and immunotherapy. Finally, the external validation of the nomograms did not conduct, thus we could 
not verify the validity of the nomograms. Hence, further clinical studies are needed to explore prognostic factors more comprehensively and validate our survival nomogram for elderly patients with osteosarcoma.

\section{Conclusions}

In conclusion, age, SEER summary stage, AJCC stage, surgery for primary site, chemotherapy, and tumor grade are independent prognostic factors of OS while age, SEER summary stage, AJCC stage, surgery for primary site, chemotherapy, and $\mathrm{M}$ stage are independent prognostic factors of CSS for an elderly patient with osteosarcoma. Our survival nomogram models provide an applicable tool with good discrimination and calibration abilities to predict the prognosis of an elderly patient with osteosarcoma.

\section{Data Sharing Statement}

All data in our study are available from the corresponding author upon reasonable request.

\section{Ethics Approval}

The authors are accountable for all aspects of the work in ensuring that questions related to the accuracy or integrity of any part of the work are appropriately investigated and resolved. The study was conducted in accordance with the Declaration of Helsinki (as revised in 2013). All information from the SEER program is available and free for public and approval was obtained from the Ethics Committee of Anyue County People's Hospital.

\section{Author Contributions}

All authors contributed to data analysis, drafting or revising the article, have agreed on the journal to which the article will be submitted, gave final approval of the version to be published, and agree to be accountable for all aspects of the work.

\section{Funding}

This work was supported by a grant from the Ziyang Science and Technology Project (grant no. Zykjjs202018-33).

\section{Disclosure}

The authors have no conflicts of interest to declare.

\section{References}

1. Mirabello L, Troisi RJ, Savage SA. International osteosarcoma incidence patterns in children and adolescents, middle ages and elderly persons. Int J Cancer. 2009;125:229-234. doi:10.1002/ijc.24320
2. Kager L, Tamamyan G, Bielack S. Novel insights and therapeutic interventions for pediatric osteosarcoma. Future Oncol. 2017;13:357-368. doi:10.2217/fon-2016-0261

3. Simpson E, Brown HL. Understanding osteosarcomas. JAAPA. 2018;31:15-19. doi:10.1097/01.JAA.0000541477.24116.8d

4. Khandekar S, Dive A, Munde P, Fande PZ. Chondroblastic osteosarcoma of the left zygomatic bone: rare case report and review of the literature. J Oral Maxillofac Pathol. 2014;18:281-285. doi:10.4103/ 0973-029X.140791

5. Bacci G, Bertoni F, Longhi A, et al. Neoadjuvant chemotherapy for high-grade central osteosarcoma of the extremity. Histologic response to preoperative chemotherapy correlates with histologic subtype of the tumor. Cancer-Am Cancer Soc. 2003;97:3068-3075.

6. Gao Z, Zhou S, Song H, Wang Y, He X. Nomograms for predicting overall survival and cancer-specific survival of chondroblastic osteosarcoma patients. J Surg Oncol. 2020;122:1676-1684. doi:10.1002/ jso. 26185

7. Tempelaere C, Biau D, Babinet A, Anract P. Osteosarcoma after the age of fifty: a clinicopathological study. Eur J Surg Oncol. 2019;45:1288-1292. doi:10.1016/j.ejso.2019.04.010

8. Tsuchie H, Emori M, Nagasawa H, et al. Prognosis of primary osteosarcoma in elderly patients: a comparison between young and elderly patients. Med Princ Pract. 2019;28:425-431. doi:10.1159/ 000500404

9. Wang Z, Wu B, Zhou Y, et al. Predictors of the survival of primary and secondary older osteosarcoma patients. $J$ Cancer. 2019;10:4614-4622. doi:10.7150/jca.32627

10. Iasonos A, Schrag D, Raj GV, Panageas KS. How to build and interpret a nomogram for cancer prognosis. $J$ Clin Oncol. 2008;26:1364-1370. doi:10.1200/JCO.2007.12.9791

11. He Y, Liu H, Wang S, Zhang J. A nomogram for predicting cancer-specific survival in patients with osteosarcoma as secondary malignancy. Sci Rep. 2020;10:12817. doi:10.1038/s41598-02069740-2

12. Chen H, Liu J, Cheng Z, et al. Development and external validation of an MRI-based radiomics nomogram for pretreatment prediction for early relapse in osteosarcoma: a retrospective multicenter study. Eur J Radiol. 2020;129:109066. doi:10.1016/j.ejrad.2020.109066

13. Wu G, Zhang M. A novel risk score model based on eight genes and a nomogram for predicting overall survival of patients with osteosarcoma. BMC Cancer. 2020;20:456. doi:10.1186/s12885-020-06741-4

14. Zhang J, Xu J, Jin S, Gao W, Guo R, Chen L. The development and validation of a nomogram for predicting brain metastases in lung squamous cell carcinoma patients: an analysis of the Surveillance, Epidemiology, and End Results (SEER) database. J Thorac Dis. 2021;13:270-281. doi:10.21037/jtd-20-3494

15. Zhu X, Huang R, Hu P, et al. Prognostic factors for survival in patients with malignant giant cell tumor of bone: a risk nomogram analysis based on the population. Med Sci Monit. 2021;27:e929154.

16. Battersby NJ, Bouliotis G, Emmertsen KJ, et al. Development and external validation of a nomogram and online tool to predict bowel dysfunction following restorative rectal cancer resection: the POLARS score. Gut. 2018;67:688-696.

17. Pan X, Yang W, Chen Y, Tong L, Li C, Li H. Nomogram for predicting the overall survival of patients with inflammatory breast cancer: a SEER-based study. Breast. 2019;47:56-61. doi:10.1016/j. breast.2019.05.015

18. Xiong Y, Cao H, Zhang Y, et al. Nomogram-predicted survival of breast cancer brain metastasis: a SEER-based population study. World Neurosurg. 2019;128:e823-e834. doi:10.1016/j.wneu.2019.04.262

19. Yu C, Zhang Y. Development and validation of prognostic nomogram for young patients with gastric cancer. Ann Transl Med. 2019;7:641. doi:10.21037/atm.2019.10.77

20. Chen S, Liu Y, Yang J, et al. Development and validation of a nomogram for predicting survival in male patients with breast cancer. Front Oncol. 2019;9:361. doi:10.3389/fonc.2019.00361 
21. Sun HH, Chen XY, Cui JQ, Zhou ZM, Guo KJ. Prognostic factors to survival of patients with chondroblastic osteosarcoma. Medicine. 2018;97:e12636. doi:10.1097/MD.0000000000012636

22. Eric I, Petek EA, Koprivcic I, Babic M, Pacaric S, Trogrlic B. Independent factors FOR poor prognosis in young patients with stage I-III breast cancer. Acta Clin Croat. 2020;59:242-251.

23. Lian W, Fu F, Lin Y, et al. The impact of young age for prognosis by subtype in women with early breast cancer. Sci Rep. 2017;7:11625. doi:10.1038/s41598-017-10414-x

24. Hagleitner MM, Hoogerbrugge PM, van der Graaf WT, et al. Age as prognostic factor in patients with osteosarcoma. Bone. 2011;49:1173-1177. doi:10.1016/j.bone.2011.08.014

25. Isakoff MS, Bielack SS, Meltzer P, Gorlick R. Osteosarcoma: current treatment and a collaborative pathway to success. J Clin Oncol. 2015;33:3029-3035. doi:10.1200/JCO.2014.59.4895

26. Janeway KA, Barkauskas DA, Krailo MD, et al. Outcome for adolescent and young adult patients with osteosarcoma: a report from the Children's Oncology Group. Cancer-Am Cancer Soc. 2012;118:4597-4605.

27. Bielack SS, Kempf-Bielack B, Delling G, et al. Prognostic factors in high-grade osteosarcoma of the extremities or trunk: an analysis of 1702 patients treated on neoadjuvant cooperative osteosarcoma study group protocols. J Clin Oncol. 2002;20:776-790. doi:10.1200/ JCO.2002.20.3.776

28. Iwata S, Ishii T, Kawai A, et al. Prognostic factors in elderly osteosarcoma patients: a multi-institutional retrospective study of 86 cases. Ann Surg Oncol. 2014;21:263-268. doi:10.1245/s10434-013-3210-4
29. Manoso MW, Healey JH, Boland PJ, et al. De novo osteogenic sarcoma in patients older than forty: benefit of multimodality therapy. Clin Orthop Relat Res. 2005;438:110-115. doi:10.1097/01. blo.0000179587.42350.4d

30. Nishida Y, Isu K, Ueda T, et al. Osteosarcoma in the elderly over 60 years: a multicenter study by the Japanese Musculoskeletal Oncology Group. J Surg Oncol. 2009;100:48-54. doi:10.1002/jso.21287

31. Fan XL, Cai GP, Zhu LL, Ding GM. Efficacy and safety of ifosfamide-based chemotherapy for osteosarcoma: a meta-analysis. Drug Des Devel Ther. 2015;9:5925-5932. doi:10.2147/DDDT. S91217

32. Jiang Y, Wang T, Wei Z. Construction and validation of nomograms for predicting the prognosis of juvenile osteosarcoma: a real-world analysis in the SEER database. Technol Cancer Res Treat. 2020;19:1079215366. doi:10.1177/1533033820947718

33. Hayakawa K, Matsumoto S, Ae K, et al. Definitive surgery of primary lesion should be prioritized over preoperative chemotherapy to treat high-grade osteosarcoma in patients aged 41-65 years. J Orthop Traumatol. 2020;21:13. doi:10.1186/s10195-020-00552-w

34. Grimer RJ, Cannon SR, Taminiau AM, et al. Osteosarcoma over the age of forty. Eur J Cancer. 2003;39:157-163. doi:10.1016/S09598049(02)00478-1
International Journal of General Medicine

\section{Publish your work in this journal}

The International Journal of General Medicine is an international, peer-reviewed open-access journal that focuses on general and internal medicine, pathogenesis, epidemiology, diagnosis, monitoring and treatment protocols. The journal is characterized by the rapid reporting of reviews, original research and clinical studies

\section{Dovepress}

across all disease areas. The manuscript management system is completely online and includes a very quick and fair peer-review system, which is all easy to use. Visit http://www.dovepress.com/ testimonials.php to read real quotes from published authors. 